\title{
Model of a multiverse providing the dark energy of our universe ${ }^{\mathrm{a}}$
}

\author{
E. Rebhan ${ }^{\mathrm{b}}$ \\ Institut für Theoretische Physik, \\ Heinrich-Heine-Universität, \\ D-40225 Düsseldorf, Germany
}

It is shown that the dark energy presently observed in our universe can be regarded as the energy of a scalar field driving an inflation-like expansion of a multiverse with ours being a subuniverse among other parallel universes. A simple model of this multiverse is elaborated: Assuming closed space geometry, the origin of the multiverse can be explained by quantum tunneling from nothing; subuniverses are supposed to emerge from local fluctuations of separate inflation fields. The standard concept of tunneling from nothing is extended to the effect that in addition to an inflationary scalar field, matter is also generated, and that the tunneling leads to an (unstable) equilibrium state. The cosmological principle is assumed to pertain from the origin of the multiverse until the first subuniverses emerge. With increasing age of the multiverse, its spatial curvature decays exponentially so fast that, due to sharing the same space, the flatness problem of our universe resolves by itself. The dark energy density imprinted by the multiverse on our universe is time-dependent, but such that the ratio $w=\varrho /\left(c^{2} p\right)$ of its mass density and pressure (times $\left.c^{2}\right)$ is timeindependent and assumes a value $-1+\epsilon$ with arbitrary $\epsilon>0 . \epsilon$ can be chosen so small, that the dark energy model of this paper can be fitted to the current observational data as well as the cosmological constant model.

PACS numbers: 98., 98.80.-k, 98.80.Bp, 98.80.Cq, 98.80.Qc

Keywords: multiverse, dark energy, creation out of nothing

\footnotetext{
${ }^{a}$ Preprint of an article published in Int. J. Mod. Phys. A, Vol. 32, No. 25 (2017) 1750149 (29 pages), https://doi.org/10.1142/S0217751X17501494 (C) copyright World Scientific Publishing Company, http://www.worldscientific.com/worldscinet/ijmpa.

b Eckhard.Rebhan@uni-duesseldorf.de
} 


\section{INTRODUCTION}

The concept of dark energy (DE) was introduced in order to fill a gap in the present energy content of our universe and to explain the presently observed acceleration of its expansion [1]. Best agreement between observations and the predictions of the standard model of cosmology is obtained by assuming that DE contributes $68,3 \%$ of the total energy in the observable universe. The oldest and best known models for its theoretical treatment are its representation by a cosmological constant or by a dynamical scalar field, sometimes called quintessence (see e.g. Ref. [2] and references therein). Numerous further models were proposed, and still many more papers on DE have been published. According to Ref. [3], the proposed models can be categorized into eight different groups, applications of the holographic principle and back-reaction of gravity being more recent ones. In spite of all these efforts, the true nature of DE is not yet unveiled.

A scalar field used for describing cosmic inflation, called inflaton, is of similar kind (obeys the same equations) as the scalar fields used for modeling DE, save that it is much stronger. On the one hand, inflation has, for many reasons, become an indispensable supplement to the model for any kind of expanding universe. On the other hand, important as it may be for our universe, DE seems to be no necessary ingredient of a universe in general. In other words, DE is present in our universe, but it is not clear why. This paper attempts to give an answer to this question and therefore it cannot be classified among the above mentioned categories in an important aspect. Technically, it describes the DE observed in our universe by a scalar field. The energy of the latter is shown to be identifiable with that of a scalar field driving the inflation-like expansion of an all-embracing and much older multiverse with ours being a subuniverse among others. Thus, the answer to the above question is that our DE constitutes the fingerprint of a superordinated multiverse generated by this energy.

The notion of a cosmic multiverse came up in the context of eternal inflation, introduced by Steinhardt in Ref. [4] and by Vilenkin [5]. Thereby, the multiverse emerges as a byproduct of the evolution of our universe from an inflaton field by inflationary expansion and subsequent decay of the latter; actually, the decay does not occur simultaneously everywhere, but at different times in different places. The continuously growing number of subuniverses thus produced is surrounded by rapidly growing regions of still inflating space.

The approach of this paper is different in such a way that the multiverse is considered 
as an independent entity, sustained by a scalar inflation field $\Phi$ of its own which gives rise to a permanent inflation-like expansion driven by an appropriately chosen potential $V(\Phi)$. Within the multiverse, subuniverses with ours among them are supposed to emerge from local fluctuations of separate inflation fields, and for simplicity, it is assumed that as in hybrid inflation (see Ref. [6]), they do not inflate eternally. (For better distinction from the latter and due to its - further established - identification with the DE of our universe, $\Phi$ is called DE field in this paper.) Furthermore, we assume that the multiverse lives in curved closed space. Thus, similar to the case of de Sitter space elaborated by Vilenkin [7, 8], its creation out of nothing becomes possible. An additional motivation for assuming spatial closeness, based on a not quite obvious but profound difference between closed and open space, is presented in Appendix A. ${ }^{1}$ For the sake of simplicity we assume that the closed multiverses considered satisfy the cosmological principle from their origin until the first subuniverses emerge.

A closed space-time with Friedmann Robertson Walker (FRW) metric, generated by a DE field $\Phi$ alone represents a homogeneous and isotropic entity that in the following is occasionally referred to as $\Phi$-multiverse. In Section IIIC, it is shown that $\Phi$-multiverses can provide the background of inhomogeneous multiverses containing a multitude of subuniverses, what means, that $\Phi$ keeps to be the only cosmic substrate and remains unaffected (all internal symmetries being maintained) within all space between the gradually emerging subuniverses. Due to this comprehensive role of the $\Phi$-multiverses, an important task of the present paper consists in deriving suitable solutions for them (Section II).

The property of never-ending inflation-like expansion of the multiverse requested above can be achieved by assuming that the potential $V(\Phi)$ decreases monotonically with increasing $\Phi$ for all values of $\Phi$. Since then, there is no minimum of $V(\Phi)$ around which the field $\Phi$ could oscillate, a decay of the field $\Phi$ by phase transition, triggered by field oscillations (see e.g. p. 244 of Ref. [9]), is avoided.

Simple as it may look at first glance, the multiverse concept outlined above has many implications and requires a multitude of calculations for establishing a consistent model. The following requirements must be satisfied (the sections where care is taken of them being

${ }^{1}$ A similar model of an expanding multiverse with flat spatial geometry would be possible, but appear less meaningful, because for the reason of causal connectivity, the multiverse could only cover a finite region of the infinitely extended space-time. A creation out of nothing would be impossible, and the argument of Appendix A would not apply. 
noted in brackets):

1. $\Phi$ fulfills the general relativistic equations for a scalar quantum field, driven by a potential $V(\Phi)$ which prevents oscillations of $\Phi(T)$ and causes a continuously accelerated inflation-like expansion (Section II).

2. The DE presently observed in our universe can be attributed to the field $\Phi$. For that, the mass density $\varrho_{\Phi}$ of $\Phi$ must not only have the proper present value (in Section III A, it is derived how it transfers to our universe), but must also drive the accelerated expansion presently observed in our universe (Section III B). Furthermore it may not be space-dependent there (Section III A).

3. The initial value of $\varrho_{\Phi}$ may not exceed the Planck density $\varrho_{P}$ (Section II A 1 ).

4. Our universe fits into the multiverse, not only spatially but also time-wise (Section II A 2).

5. The present curvature of the multiverse is so small that it lies well below the present limits of measurability (Section II A 2).

6. The dynamics of the expansion can be arranged in such a way that the initial state can be explained to come about by quantum tunneling from nothing (Section II B 2).

7. The properties of our universe and of the DE following from our model agree with those predicted by the standard model of cosmology for a practically uncurved space and are fitting the current observational data (Section III B and IV).

Since the number of model parameters is quite small for a fitting, it is by no means evident that all requirements can be satisfied.

\section{EVOLUTION OF PURE $\Phi$-MULTIVERSES}

In this section, we determine the evolution of $\Phi$-multiverses ignoring the presence of subuniverses. According to Section III, in a multiverse with subuniverses this yields the correct result for all regions outside the latter ones. All calculations are carried out in FRW 
coordinates. The basic equations to be satisfied in a closed multiverse with positive spatial curvature are (see, e.g., Ref. [10] or p. 550 in Ref. [11]) ${ }^{2}$

$$
\begin{gathered}
H^{2}=\frac{\dot{A}^{2}(T)}{A^{2}(T)}=\frac{8 \pi G}{3} \varrho-\frac{c^{2}}{A^{2}} \quad \text { with } \quad \varrho=\varrho_{m}+\varrho_{\Phi}, \\
\varrho_{\Phi}=\frac{\hbar^{2} \dot{\Phi}^{2}(T)}{2 \mu c^{4}}+\frac{V(\Phi)}{c^{2}}, \quad p_{\Phi}=\frac{\hbar^{2} \dot{\Phi}^{2}(T)}{2 \mu c^{2}}-V(\Phi), \\
\ddot{\Phi}(T)+3 H \dot{\Phi}(T)+\frac{\mu c^{2}}{\hbar^{2}} V^{\prime}(\Phi)=0 .
\end{gathered}
$$

For better discriminability, the cosmic scale factor and the time are denoted by $A$ and $T$ in a multiverse and by $a$ and $t$ in subuniverses. $\varrho_{m}$ and $\varrho_{\Phi}$ are the mass densities of matter and the DE field $\Phi$, respectively, and $\mu$ is the mass parameter of the field $\Phi$.

For the evaluation of Eqs. (1)-(3), it turns out useful to introduce relative quantities, and to do this separately for the very early and the later evolution of the multiverse; furthermore, we admit the possibility that initially besides the DE field, matter is also present. Since the present value of $\varrho_{\Phi}$ plays a decisive role, we begin with the later evolution.

\section{A. Later evolution}

\section{General theory}

The relative quantities used for the later evolution are

$$
x=A / A_{0}, \quad \tau=T / t_{H 0},
$$

the index zero referring to the present values, i.e. $A_{0}=A\left(T_{0}\right)$ etc. and $x=1$ for $T=T_{0}$.

$$
t_{H 0}=\sqrt{\frac{3}{8 \pi G \varrho_{c 0}}}=\frac{1}{H_{0}}=14.0 \cdot 10^{9} \mathrm{a}=4.41 \cdot 10^{17} \mathrm{~s}
$$

and

$$
\varrho_{c 0}=\frac{3 H_{0}^{2}}{8 \pi G}=9.20 \cdot 10^{-27} \mathrm{~kg} \mathrm{~m}^{-3}
$$

are the present Hubble time and the critical density of our universe respectively for the Hubble parameter $H_{0}=70 \mathrm{~km} \mathrm{~s}^{-1} / \mathrm{Mpc}$. Considering the densities as functions of $x$ we set

$$
\varrho=\varrho_{\Phi 0} g(x) \quad \text { with } \quad g(x)=\frac{\varrho_{m}\left(A_{0} x\right)+\varrho_{\Phi}\left(A_{0} x\right)}{\varrho_{\Phi 0}} .
$$

\footnotetext{
${ }^{2}$ In order to make numerical evaluations more transparent, in this paper MSI units are used.

${ }^{3}$ Latest measurements yielded $H_{0}=67.6_{-0.6}^{+0.7}$ (SDSS-III Baryon Oscillation Spectroscopic Survey data from 07.13.2016) and $H_{0}=71.9_{-3.0}^{+2.4}$ (Hubble Space Telescope data from 11.22.2016).
} 
According to the later Eq. (67), $\varrho_{\Phi 0}$ can be identified directly with the mass density $0.683 \varrho_{c 0}$ of DE presently observed in our universe, i.e.

$$
\varrho_{\Phi 0}=\varrho_{\Phi}\left(A_{0}\right)=0.683 \varrho_{c 0}=6.28 \cdot 10^{-27} \mathrm{~kg} \mathrm{~m}^{-3} .
$$

Inserting Eqs. (4)-(7) in Eq. (1) yields

$$
\dot{x}^{2}(\tau)=\frac{\varrho_{\Phi 0}}{\varrho_{c 0}}\left(x^{2} g(x)-\frac{3 c^{2}}{8 \pi G \varrho_{\Phi 0} A_{0}^{2}}\right) .
$$

Since our universe must fit into the multiverse, $A_{0} \gg R$ must hold where

$$
R=a\left(t_{0}\right) r_{b o}=23.5 \cdot 10^{9} \mathrm{ly}=2.22 \cdot 10^{26} \mathrm{~m}
$$

is the present metric radius of the boundary of our observable universe (at radial coordinate $r_{b o}$ and for scale factor $a\left(t_{0}\right)$ reached at present time $\left.t_{0}\right)$. Accordingly, the second term in the brackets is very small, and it turns out, that in all interesting cases, the bracket has a zero at some $x=x_{*}<1$. This zero is admissible, if the physical solution is prevented from running into it. The latter can be achieved by requiring $x_{*} \leq x_{i}$, where $x_{i}$ is the initial value from which the expansion of the multiverse starts. We decide for the choice $x_{*}=x_{i}$ or

$$
x_{i}^{2} g\left(x_{i}\right)=\frac{3 c^{2}}{8 \pi G \varrho_{\Phi 0} A_{0}^{2}},
$$

which causes the multiverse to start with zero expansion velocity,

$$
\dot{x}(\tau)=0 \quad \text { for } \quad x=x_{i},
$$

and enables its creation out of nothing by quantum tunneling (see Subsection II B 2). With this and Eq. (8), Eq. (9) finally yields

$$
\dot{x}(\tau)=0.826 \sqrt{x^{2} g(x)-x_{i}^{2} g\left(x_{i}\right)}
$$

and

$$
\tau(x)=1.21 \int_{x_{i}}^{x} \frac{d \xi}{\sqrt{\xi^{2} g(\xi)-x_{i}^{2} g\left(x_{i}\right)}} \quad \text { for } \quad \tau\left(x_{i}\right)=0 .
$$

We assume that the expansion of the multiverse starts from the scale factor $A_{i}=l_{P}$, where

$$
l_{P}=\sqrt{\frac{\hbar G}{c^{3}}}=1.616 \cdot 10^{-35} \mathrm{~m}
$$

is the Plank length, whence we have

$$
x_{i}=\frac{l_{P}}{A_{0}}=\frac{l_{P}}{R \zeta} \quad \text { with } \quad \zeta:=\frac{A_{0}}{R} .
$$


(In Section IV consequences of the more general condition $A_{i}=\lambda l_{P}$ with $\lambda \geq 1$ are discussed.) Inserting this in Eq. (11) yields

$$
g_{i}=g\left(x_{i}\right)=\frac{3 c^{2}}{8 \pi G \varrho_{\Phi 0} l_{P}^{2}}=9.80 \cdot 10^{121} \quad \text { and } \quad \zeta=\frac{l_{P}}{R x\left(g_{i}\right)}=\frac{7.28 \cdot 10^{-62}}{x_{i}} .
$$

(For evaluation $c=2.998 \cdot 10^{8} \mathrm{~ms}^{-1}, G=6.673 \cdot 10^{-11} \mathrm{~m}^{3} \mathrm{~kg}^{-1} \mathrm{~s}^{-2}$ and Eqs. (8), (10) and (15) were used; $x(g)$ is the inverse of the function $g(x)$.) Imposing on $g(x)$ the condition

$$
d\left[x^{2} g(x)\right] / d x>0 \quad \text { for all } x,
$$

according to Eq. (13) we achieve a continuously accelerated expansion of the multiverse that with growing $x$ approaches an inflation-like state.

For checking the fulfillment of requirement 3 of the Introduction, the initial density $\varrho_{i}$ must be calculated using the initial condition (12) or $\dot{A}(T)=0$ for $A=A_{i}=l_{P}$. For this, we obtain from Eqs. (1), (7a) and (15)

$$
\varrho_{i}=\varrho_{m i}+\varrho_{\Phi i}=\frac{3 c^{2}}{8 \pi G l_{P}^{2}}=\frac{3}{8 \pi} \varrho_{P}=\varrho_{\Phi 0} g_{i}
$$

where at last the definition

$$
\varrho_{P}=\frac{c^{5}}{\hbar G^{2}}=5.157 \cdot 10^{96} \mathrm{~kg} \mathrm{~m}^{-3}
$$

of the Planck density $\varrho_{P}$ was used. $\varrho_{i}$ is fixed to a value slightly below the Planck density as demanded. This result is completely independent of the composition and later behavior of the density $\varrho$ and is solely due to the vanishing of the initial expansion velocity.

We now turn to solving Eqs. (2)-(3). Inserting $\varrho_{\Phi}=\varrho_{\Phi}(A)$ in Eq. $(2 a)^{4}$ and deriving it with respect to $T$ yields

$$
\dot{\Phi}(T)\left(\frac{\hbar^{2} \ddot{\Phi}(T)}{\mu c^{4}}+\frac{V^{\prime}(\Phi)}{c^{2}}\right)=\varrho_{\Phi}^{\prime}(A) \dot{A}(T) .
$$

Inserting in this $\ddot{\Phi}(T)$ from Eq. (3) and $H=\dot{A}(T) / A$, after some rearrangement we obtain

$$
\dot{\Phi}(T)= \pm \frac{c^{2}}{\hbar} \sqrt{-\frac{\mu A \varrho_{\Phi}^{\prime}(A)}{3}} .
$$

Note that this equation holds independent of whether or not $\varrho_{m} \equiv 0$. From it follows the conditions $\varrho_{\Phi}^{\prime}(A) \leq 0$. Since according to condition (1) of the Introduction $\Phi(T)$ may not oscillate, the possibility $\dot{\Phi}(T)=0$ must be excluded whence

$$
\varrho_{\Phi}^{\prime}(A)<0 \quad \text { and } \quad \dot{\Phi}(T)=\frac{c^{2}}{\hbar} \sqrt{-\frac{\mu A \varrho_{\Phi}^{\prime}(A)}{3}} .
$$

\footnotetext{
${ }^{4}$ Eq. (2a) is supposed to denote the first of the Eqs. (2), Eq. (2b) the second etc..
} 
(Due to $\dot{\Phi}(T) \neq 0$, the minus branch of $\dot{\Phi}(T)$ displayed in Eq. (22) can be excluded.) Introducing $x$ and $\tau$ from Eqs. (4) in Eq. (23) yields

$$
\dot{\Phi}(\tau)=\delta \sqrt{-x f^{\prime}(x)} \quad \text { where } \quad f(x)=\frac{\varrho_{\Phi}\left(A_{0} x\right)}{\varrho_{\Phi 0}}, \quad \delta=\frac{c^{2} t_{H 0} \sqrt{\mu \varrho_{\Phi 0}}}{\sqrt{3} \hbar} .
$$

From Eqs. (13) and (24a), we get

$$
\frac{d \Phi}{d x}=\frac{\dot{\Phi}(\tau)}{\dot{x}(\tau)}=\frac{1.21 \delta \sqrt{-x f^{\prime}(x)}}{\sqrt{x^{2} g(x)-x_{i}^{2} g\left(x_{i}\right)}} \quad \text { or } \quad \Phi(x)=1.21 \delta \int_{x_{i}}^{x} \frac{\sqrt{-\xi f^{\prime}(\xi)} d \xi}{\sqrt{\xi^{2} g(\xi)-x_{i}^{2} g\left(x_{i}\right)}}
$$

for the initial condition $\Phi\left(x_{i}\right)=0$. Inserting Eqs. (22) and (24b) in Eq. (2a) yields

$$
\frac{V(\Phi)}{\varrho_{\Phi 0} c^{2}}=\left[f(x)+\frac{x f^{\prime}(x)}{6}\right]_{x=x(\Phi)}
$$

where $x(\Phi)$ is the inverse function of $\Phi(x)$. Although Eq. (3) was used in deriving Eq. (26), we must still make sure that it is actually satisfied. According to Eqs. (21) and (1) we have

$$
\ddot{\Phi}(T)+\frac{\mu c^{2}}{\hbar^{2}} V^{\prime}(\Phi)=\frac{\mu c^{4} A \varrho_{\Phi}^{\prime}(A)}{\hbar^{2}} \frac{H}{\dot{\Phi}(T)}=-3 H \dot{\Phi}(T),
$$

where at last the square of Eq. (22) was used. This confirms the fulfillment of Eq. (3) and reveals together with the result (26) that the field $\Phi$ has the structure required by the usual theory of scalar fields (requirement 1 of the Introduction).

\section{Specialization to $\varrho_{\Phi} \sim x^{\gamma-2}$}

Due to our assumption that the field $\Phi$ does not decay into regular matter by a phase transition, we must only account for initially present mass of density $\varrho_{m}$ obeying the equation of state

$$
\varrho_{m}=\frac{\varrho_{m i} A_{i}^{n}}{A^{n}}=\frac{\varrho_{m i} x_{i}^{n}}{x^{n}},
$$

where $n=3$ for cold and $n=4$ for hot matter. Concerning an appropriate ansatz for $\varrho_{\Phi}$, we have to observe the inequality $(23 \mathrm{a})$ or $g^{\prime}(x)<0$, and the inequality (18), according to which $g(x)$ must decrease more slowly than $\hat{g}(x)=C / x^{2}$ with increasing $x$ since $d\left[x^{2} \hat{g}(x)\right] / d x=0$. Due to this and the validity of Eq. (29a) for almost all $x$, we employ the ansatz

$$
\varrho_{\Phi}=\varrho_{\Phi 0} x^{\gamma-2} \quad \text { or } \quad f(x)=x^{\gamma-2} \quad \text { with } \quad 0<\gamma<2
$$

Since the spatial curvature of the $\Phi$-multiverse is $K=1 / A^{2} \sim x^{-2}$, we have $\varrho_{\Phi} \sim K^{1-\gamma / 2}$. This means, that the mass density $\varrho_{\Phi}$ of the field $\Phi$ is coupled to the curvature $K$ in a monotonic 
way such, that it decreases and approaches to zero together with the latter. This property goes well with the considerations of $\mathrm{A}$, according to which in a closed multiverse of positive curvature an intrinsic expansion of space should exist, not present in an open multiverse.

Restricting the initial value of $\varrho_{m}$ to the value specified in Eq. (46b) and denoting the corresponding initial value of $x$ by $x_{i m}$, we get from Eqs. (27) and (28a)

$$
\frac{\varrho_{m}}{\varrho_{\Phi}}=\frac{\gamma}{n-2}\left(\frac{x_{i m}}{x}\right)^{n+\gamma-2} \ll 1 \quad \text { for } \quad x \gg x_{i m}\left(\frac{\gamma}{n-2}\right)^{\frac{1}{n+\gamma-2}}=x_{i m} \cdot \mathcal{O}(1) .
$$

For example, for $\gamma=1.95$ and $n=3$ from Eqs. (32a) and (33a), we get $x_{i m}=\mathcal{O}(1) \cdot 10^{-2431}$. It can be concluded from this, that for almost the entire later evolution $\varrho_{m}$ can be neglected, whence according to Eqs. (7a) and (24b) we get

$$
g(x)=f(x) \quad \text { and } \quad f(1)=1
$$

in other words the later evolution is practically the same for the cases with and without primordial matter. With Eq. (28) and this, Eqs. (13)-(14) become

$$
\dot{x}(\tau)=0.826 \sqrt{x^{\gamma}-x_{i}^{\gamma}}
$$

and

$$
\tau(x)=1.21 \int_{x_{i}}^{x} \frac{d \xi}{\sqrt{\xi^{\gamma}-x_{i}^{\gamma}}}=-\frac{1.21 x \sqrt{x^{\gamma}-x_{i}^{\gamma}}}{x_{i}^{\gamma}}{ }_{2} F_{1}\left(1, \frac{2+\gamma}{2 \gamma} ; \frac{1+\gamma}{\gamma} ; \frac{x^{\gamma}}{x_{i}^{\gamma}}\right),
$$

where ${ }_{2} F_{1}(a, b ; c ; z)$ is the hypergeometric function. The quantities $x_{i}$ and $\zeta$, for $\varrho_{m} \neq 0$ and restriction to the special case of Eq. (46b) denoted by $x_{i m}$ and $\zeta_{m}$, follow from Eqs. (17) with the use of Eqs. (7) and (28a) and are

$$
\begin{array}{lll}
x_{i}=x\left(g_{i}\right)=g_{i}^{-\frac{1}{2-\gamma}}, & \zeta=\mathrm{e}^{-140.775+\frac{280.895}{2-\gamma}} \quad \text { for } \varrho_{m i}=0, \\
x_{i m}=x_{i}\left(\frac{n+\gamma-2}{n-2}\right)^{\frac{1}{2-\gamma}}, & \zeta_{m}=\zeta\left(\frac{n-2}{n+\gamma-2}\right)^{\frac{1}{2-\gamma}} \text { for } \varrho_{m i}=\frac{\gamma \varrho_{\Phi i}}{(n-2)}
\end{array}
$$

with $g_{i}$ given by Eq. (17a). A very good approximation to the result (31) is obtained by expanding the integral in Eq. (31) with respect to $u^{-\gamma / 2}$ where $u=\xi / x_{i}$ :

with

$$
\begin{gathered}
\frac{\tau(x)}{1.21}=x_{i}^{1-\frac{\gamma}{2}} \int_{1}^{\frac{x}{x_{i}}} \frac{u^{-\frac{\gamma}{2}} d u}{\sqrt{1-u^{-\gamma}}}=x_{i}^{1-\frac{\gamma}{2}} \int_{1}^{\frac{x}{x_{i}}}\left(u^{-\frac{\gamma}{2}}+\frac{u^{-\frac{3 \gamma}{2}}}{2}+\frac{3 u^{-\frac{5 \gamma}{2}}}{8}+\ldots\right) d u \\
=\frac{x^{1-\frac{\gamma}{2}}}{1-\gamma / 2}-Z(\gamma) x_{i}^{1-\frac{\gamma}{2}}+\mathcal{O}\left(x_{i}^{\gamma}\right) \\
Z(\gamma)=\frac{2}{2-\gamma}+\frac{1}{2-3 \gamma}+\frac{3}{4(2-5 \gamma)}+\ldots
\end{gathered}
$$


TABLE I. Parameters of the late multiverse as functions of $\gamma$ for $n=4$.

\begin{tabular}{l|cccccc}
\hline \hline$\gamma$ & 0.1 & 1.0 & 1.8 & 1.9 & 1.95 & 1.99 \\
\hline$\tau_{0}$ & 1.27 & 2.42 & 12.1 & 24.2 & 48.4 & 242 \\
$\zeta$ & $1.2 \cdot 10^{3}$ & $7.1 \cdot 10^{60}$ & $6.6 \cdot 10^{548}$ & $5.9 \cdot 10^{1158}$ & $4.9 \cdot 10^{2378}$ & $9.6 \cdot 10^{12137}$ \\
$\zeta_{m}$ & $1.1 \cdot 10^{3}$ & $4.8 \cdot 10^{60}$ & $2.7 \cdot 10^{547}$ & $7.5 \cdot 10^{1155}$ & $6.0 \cdot 10^{2372}$ & $9.7 \cdot 10^{12107}$ \\
$x_{i}$ & $6.2 \cdot 10^{-65}$ & $1.0 \cdot 10^{-122}$ & $1.1 \cdot 10^{-610}$ & $1.2 \cdot 10^{-1220}$ & $1.5 \cdot 10^{-2440}$ & $7.5 \cdot 10^{-12200}$ \\
$x_{s}$ & 0.213 & 0.354 & 0.430 & 0.437 & 0.441 & 0.443 \\
$\ddot{y}_{0} / \ddot{y}_{\Lambda}$ & -0.021 & 0.463 & 0.893 & 0.946 & 0.973 & 0.995 \\
$\varrho_{\Phi h} / \varrho_{\Phi}$ & 2.64 & 1.57 & 1.09 & 1.04 & 1.02 & 1.00 \\
\hline \hline
\end{tabular}

Due to the extreme smallness of $x_{i}$, for $x \gg[(1-\gamma / 2) Z(\gamma)]^{1 /(1-\gamma / 2)} x_{i}=\mathcal{O}(1) x_{i}$ the result $(31)$ can be replaced by

$$
\tau(x)=\tau_{0} x^{1-\gamma / 2} \quad \text { with } \quad \tau_{0}=\frac{1.21}{1-\gamma / 2}
$$

the error being negligibly small. Equivalently we have

$$
x=\left(\tau / \tau_{0}\right)^{1 /(1-\gamma / 2)} .
$$

Inserting $\gamma=2-2.42 / \tau_{0}$, obtained from Eq. (34b), in Eqs. (32b) and (33b) yields

$$
\begin{aligned}
\zeta\left(\tau_{0}\right) & =\mathrm{e}^{-140.775+116.072 \tau_{0}} \\
\zeta_{m}\left(\tau_{0}\right) & =\zeta\left(\tau_{0}\right)\left(\frac{n}{n-2}-\frac{2.42}{(n-2) \tau_{0}}\right)^{-0.413 \tau_{0}} \stackrel{\tau_{0} \gg 1}{\longrightarrow} \quad \zeta\left(\tau_{0}\right) \mathrm{e}^{-0.413 \tau_{0} \ln \left(\frac{n}{n-2}\right)} .
\end{aligned}
$$

From Eq. (36) follows the condition $\tau_{0}>140.775 / 116.072=1.21 . .$, since according to condition 4 of the Introduction and Eq. (16b), we must have $\zeta>1$ or $\zeta_{m}>1$ respectively. Due to the exponential growth with $\tau_{0}$, already for $\tau_{0} \approx 2.4$ both $\zeta$ 's reach a $10^{60}$-fold enhancement above the demanded minimum value 1 . This means that for almost all admissible ages of the multiverse its present radius $A_{0}=R \zeta$ is so huge that the present spatial curvature

$$
K_{0}=\frac{1}{A_{0}^{2}}=\frac{1}{R^{2} \zeta^{2}}
$$

is far below measurability.

In Appendic B it is shown that for $\gamma$ values close to 2, the condition (B1) for slow roll is satisfied in the whole range of validity of the later evolution. Simultaneously, the solution (40) for $V$ approaches the slow roll approximation (B2), $V(x)=\varrho_{\Phi 0} c^{2} x^{\gamma-2}=\varrho_{\Phi}(x) c^{2}$. 
In table I, the present age $\tau_{0}=\tau(1)$ of the multiverse is shown in multiples of $t_{H_{0}}$ for several values of $\gamma$ together with the corresponding values of $\zeta$ and $\zeta_{m}$ from Eqs. (32)-(33) and with further values discussed in Section III B. It is seen that for reasonable ages $\tau_{0} \gg 1$ the parameter $\gamma$ must be close to 2 .

Inserting Eqs. (28b) and (29a) in Eq. (25b) and substituting $u=\xi / x_{i}$ yields

$$
\Phi=1.21 \delta \sqrt{2-\gamma} \int_{x_{i}}^{x} \sqrt{\frac{\xi^{\gamma-2}}{\xi^{\gamma}-x_{i}^{\gamma}}} d \xi=1.21 \delta \sqrt{2-\gamma} \int_{1}^{\frac{x}{x_{i}}} \frac{d u}{u \sqrt{1-u^{-\gamma}}} .
$$

Expanding $1 / \sqrt{1-u^{-\gamma}}$ with respect to $u^{-\gamma}$ and neglecting all terms $\sim\left(x_{i} / x\right)^{n \gamma}$, for $x \gg x_{i}$, we obtain the approximate result

where

$$
\Phi=1.21 \delta \sqrt{2-\gamma}\left(\ln \frac{x}{x_{i}}+\frac{Z}{\gamma}\right) \quad \text { or } \quad x=x_{i} \exp \left(\frac{0.826 \Phi}{\delta \sqrt{2-\gamma}}-\frac{Z}{\gamma}\right)
$$

Inserting Eqs. (28b) and (39b) in Eq. (26) yields

$$
\frac{V(\Phi)}{\varrho_{\Phi 0} c^{2}}=\frac{4+\gamma}{6 x^{2-\gamma}}=\frac{4+\gamma}{6 x_{i}^{2-\gamma}} \exp \left(\frac{Z}{\gamma}-\frac{0.826 \Phi}{\delta \sqrt{2-\gamma}}\right) .
$$

\section{B. Very early evolution and creation of the multiverse}

\section{Very early evolution}

For dealing with the early evolution of $\Phi$-multiverses, we adapt our equations to the small scales involved. Instead of the relative quantities defined in Eq. (4), now we employ

$$
x=\frac{A}{l_{P}}, \quad \tau=\frac{T}{t_{P}} \quad \text { with } \quad t_{P}=\sqrt{\frac{\hbar G}{c^{5}}}=5.39 \cdot 10^{-44} \mathrm{~s},
$$

whence $x=1$ for $A=l_{P}$. Instead of the Eqs. (27) and (28) we use

$$
\varrho_{\Phi}=\varrho_{\Phi i} x^{\gamma-2}, \quad \varrho_{m}=\varrho_{m i} x^{-n}
$$

With this and the identities $l_{P} / t_{P}=c$ and $G t_{P}^{2} \varrho_{P}=1$, the latter following from the definitions (41c) and (20), Eq. (1) becomes

$$
\dot{x}(\tau)=\sqrt{\frac{8 \pi}{3 \varrho_{P}}\left(\varrho_{m i} x^{2-n}+\varrho_{\Phi i} x^{\gamma}\right)-1} .
$$


In the case $\varrho_{m i}=0$, with Eq. (20), we get $\varrho_{\Phi i}=\varrho_{i}=3 \varrho_{P} /(8 \pi)$ and Eq. (43) simplifies to

$$
\dot{x}(\tau)=\sqrt{x^{\gamma}-1} .
$$

Differentiating Eq. (43) with respect to $\tau$ yields

$$
\ddot{x}(\tau)=\frac{4 \pi\left[(2-n) \varrho_{m i} x^{2-n}+\gamma \varrho_{\Phi i} x^{\gamma}\right]}{3 \varrho_{P} x},
$$

and from this it follows, that at $x=1$ we have

$$
\ddot{x}(\tau)=0 \quad \text { for } \quad \varrho_{m i}=\frac{\gamma \varrho_{\Phi i}}{n-2} .
$$

Inserting this in Eq. (19) yields

$$
\varrho_{m i}=\frac{3 \varrho_{P}}{8 \pi} \frac{\gamma}{(n-2+\gamma)}, \quad \varrho_{\Phi i}=\frac{3 \varrho_{P}}{8 \pi} \frac{(n-2)}{(n-2+\gamma)} .
$$

For these parameters Eqs. (43) and (45) assume the form

$$
\dot{x}(\tau)=\sqrt{\frac{(n-2) x^{\gamma}+\gamma x^{2-n}}{n-2+\gamma}-1}, \quad \ddot{x}(\tau)=\frac{(n-2) \gamma}{2(n-2+\gamma)} \frac{\left(x^{\gamma}-x^{2-n}\right)}{x} .
$$

At $x=1$ the system is in an equilibrium state with respect to the variable $x(\tau)$ since according to Eqs. (48), not only $\dot{x}(\tau)=0$, but also $\ddot{x}(\tau)=\dddot{x}(\tau)=\ldots=0$. (This is different from the case without matter since in that case $\left.\ddot{x}(\tau)\right|_{x=1}=\gamma / 2$ according to Eq. (44).) Like Einsteins solution for a static universe, this equilibrium is unstable since $\ddot{x}>0$ for $x>1$ and $\ddot{x}<0$ for $x<1 .^{5}$ In contrast to the simpler cases mentioned in footnote 5, now also the dynamics of the field $\Phi$ must be determined from Eqs. (2)-(3). In the following we restrict our consideration to two special cases: 1. validity of Eq. (44) (no matter present), and 2. validity of Eq. (48) (matter initially in equilibrium with the DE). We can deal with both cases at a time by introducing the definition

$$
g(x)=\left\{\begin{array}{ccll}
x^{\gamma} & \text { for } & \varrho_{m} \equiv 0 \\
\frac{(n-2) x^{\gamma}+\gamma x^{2-n}}{n-2+\gamma} & \text { for } & \varrho_{m i}=\frac{3 \varrho_{P}}{8 \pi} \frac{\gamma}{(n-2+\gamma)} .
\end{array}\right.
$$

(Note that for $\varrho_{m i} \neq 0$, this definition differs slightly from that of Eq. (7).) According to its definition, the function $g(x)$ has the property

$$
g(1)=1
$$

5 A similar but somewhat simpler solution of the cosmological equations, also starting from an unstable equilibrium, was published by this author in the year 2000 [12]. As in Refs. [7] and [8], a constant density $\varrho_{\Phi}$, corresponding to a cosmological constant, was considered there; it was transfered into an initial equilibrium by the presence of matter as well. 
With the above definition, the joint representation of Eqs. (44) and (48a) becomes

$$
\dot{x}(\tau)=\sqrt{g(x)-1} .
$$

Using $A \varrho_{\Phi}^{\prime}(A)=x \varrho_{\Phi}^{\prime}(x)=-(2-\gamma) \varrho_{\Phi} x^{\gamma-2}$ and $\dot{\Phi}(\tau)=t_{P} \dot{\Phi}(T)$ according to Eq. (41b), we obtain from Eq. (23b)

$$
\dot{\Phi}(\tau)=\frac{t_{P} c^{2}}{\hbar} \sqrt{\frac{(2-\gamma) \mu \varrho_{\Phi i}}{3}} x^{\gamma / 2-1} .
$$

With this, Eq. (42a) and $\dot{\Phi}(T)=\dot{\Phi}(\tau) / t_{p}$ Eq. (2a) yields

$$
V(x)=\frac{(4+\gamma) \varrho_{\Phi i} c^{2}}{6 x^{2-\gamma}}
$$

and using Eqs. (51) and (52), we get

$$
\frac{d \Phi}{d x}=\frac{\dot{\Phi}(\tau)}{\dot{x}(\tau)}=\frac{t_{P} c^{2}}{\hbar} \sqrt{\frac{(2-\gamma) \mu \varrho_{\Phi i}}{3}} \frac{x^{\gamma / 2}}{x \sqrt{g(x)-1}} .
$$

At $x=1$, according to Eqs. (52) and (54) with (50), we have $\dot{\Phi}(\tau) \neq 0$ and $d x / d \Phi=0$. This means that the changes of $\Phi$ arising from $\dot{\Phi}(\tau) \neq 0$ have no influence on the (unstable) equilibrium $x \equiv 1$ and $V(x) \equiv V(1)$, or, otherwise, equilibrium exist only with respect to $x$ and $V$, but not with respect to $\Phi$ (see Fig. 4).

For non-equilibrium values $x$, integration of Eq. (54) yields

$$
\Phi(x)=\frac{t_{P} c^{2}}{\hbar} \sqrt{\frac{(2-\gamma) \mu \varrho_{\Phi i}}{3}} \int_{x_{g}}^{x} \frac{\xi^{\gamma / 2} d \xi}{\xi \sqrt{g(\xi)-1}} .
$$

In the case $\varrho_{m} \equiv 0$, in which there is no equilibrium at $x=1$, we choose $x_{g}=1$ since the integral converges although the integrand diverges like $1 / \sqrt{\xi-1}$ for $\xi \rightarrow 1$. In the case described by Eq. (47), the integrand behaves like $1 /(\xi-1)$ for $\xi \rightarrow 1$ and is not integrable. In this case, we choose $x_{g}>1$, e.g. $x_{g}=2$ in Fig. 4 .

\section{Creation of the multiverse out of nothing by quantum tunneling}

According to Refs. [7] and [8], the initial state $x=1, \dot{x}(\tau)=0$, start of the classical evolution with zero expansion velocity, can be considered as coming about by quantum-mechanical tunneling from nothing (no space, no time). An approximate quasi-classical description of the tunneling process preceding this classical high energy state can be obtained by extending equation (51) to the non-classical values $x<1$ yielding

$$
\dot{x}(\tau)=\mathrm{i} \sqrt{1-g(x)}
$$


and by employing an imaginary time

$$
\tau=-\mathrm{i} u \quad \text { with real } u,
$$

a method introduced by Coleman [13]. With this the equation of motion assumes the "Euclidean" form

$$
\dot{x}(u)=\sqrt{1-g(x)} .
$$

For $g(x)=x^{\gamma}$, we have $1-g(x)>0$ in the quantum regime $x<1$, and $\dot{x}(u)$ is well defined. The singularity of the corresponding density $\varrho_{\Phi} \sim x^{\gamma-2}$ at $x=0$ is tolerable because the total energy $E=\varrho_{\Phi} c^{2} \mathcal{V}=2 \pi c^{2} L_{P}^{3} \varrho_{\Phi i} x^{1+\gamma}$, the quantity which matters in the quantum regime, vanishes as $x \rightarrow 0$. By contrast in the case described by Eq. (48a), we would have $1-g(x)<0$ for $x<1$, and the connection to quantum tunneling becomes only possible, when a cutoff of the density $\varrho_{m}$ is introduced. (This way simultaneously a more serious singularity of $\varrho_{m}(x)$ at $x=0$ is avoided). Accordingly, in the region $x<1$, we set ${ }^{6}$

$$
\varrho_{m} \equiv \varrho_{m i} \quad \text { and } \quad g(x)=\frac{(n-2) x^{\gamma}+\gamma}{n-2+\gamma} .
$$

At $x=1$, the solution of Eq. (57) can be connected continuously with the classical solution obtained from Eq. (51) for $x \geq 1$ by putting

$$
\tau(x)=\int_{1}^{x} \frac{d \xi}{\sqrt{g(\xi)-1}}, \quad u(x)=-\int_{x}^{1} \frac{d \xi}{\sqrt{1-g(\xi)}} .
$$

Inserting Eq. (56) into the line element $d s^{2}$ yields

$$
d s^{2}=-c^{2} d u^{2}-a^{2}(u) d r^{2}+\ldots,
$$

referred to as Euclidean form. This shows that the introduction of an imaginary time amounts to converting $c t$ it into a fourth space coordinate $c u$.

In Figs. 1 and 2, it is shown for $\varrho_{m} \equiv 0, \gamma=1.9$ and for $\varrho_{m} \neq \equiv 0, n=3, \gamma=1.9$, respectively, how the expansion parameter $x$ evolves from $x=0$ in the quantum regime $x<1$ to values $x>1$. The tip at the bottom of the figures does not conform with the no boundary proposal of Hartle and Hawking [14] (nicely illustrated in Ref. [15]); on the other hand, our approach

\footnotetext{
${ }^{6}$ A cutoff at the Planck density could also be envisaged and would only marginally change the picture. However, the Plank density is a round figure only and has no precise physical explanation. The density $\varrho_{i}$ has the same order of magnitude and appears due to its physical background more appropriate for the cutoff.
} 


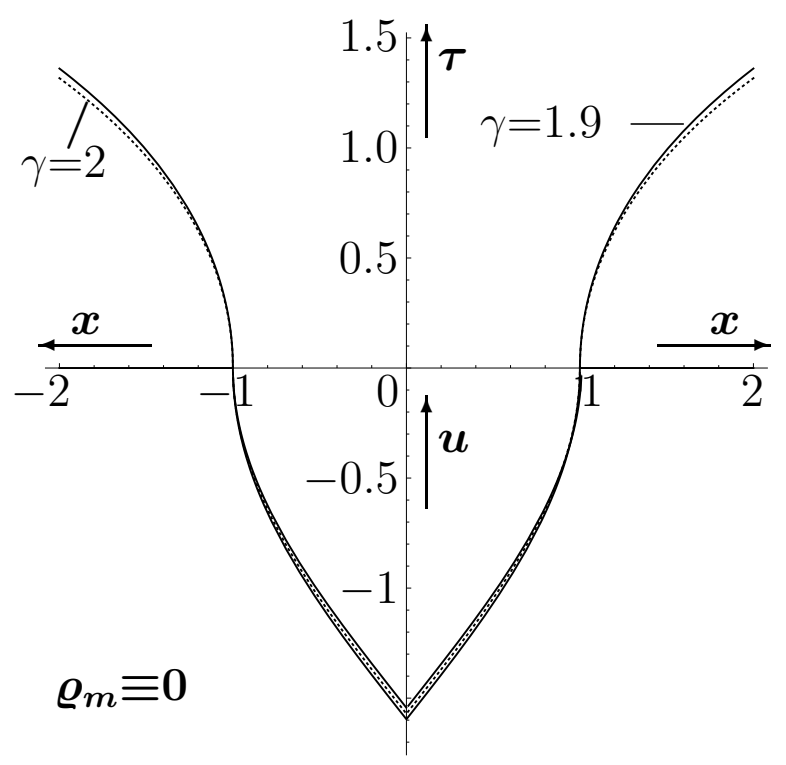

FIG. 1. Normalized age of the multiverse, $\tau$ for $x \geq 1$ and $u=\mathrm{i} \tau$ for $0 \leq x<1$, as function of the relative cosmic scale factor $x=A / l_{P}$ in the case $\varrho_{m} \equiv 0$. The mirror images of the curves $\tau(x)$ and $u(x)$ on the left-hand side express the fact that at every time $\tau$, the projection of the spherically symmetric boundary of the multiverse onto the $x, \tau$-plane yields two points symmetrically positioned with respect to the $\tau$-axis. The dotted curve obtained for $\gamma=2$ represents de Sitter space. In the quantum regime $0 \leq x<1$, the upper solid curve is obtained when the divergent density $\varrho_{\Phi}=\varrho_{\Phi i} x^{\gamma-2}$ is truncated by replacing it with $\varrho_{\Phi} \equiv \varrho_{\Phi i}$.

agrees widely with that of Vilenkin (see Refs. [7] and [8]). ${ }^{7}$ In Fig. 3, a further comparison of the case $\gamma=1.9, \varrho_{m i} \equiv 0$ with de Sitter space shows that for larger values of $x$, the differences between the two become appreciable.

In the case $\varrho_{\Phi}=$ const considered in Ref. [7], Eqs. (2)-(3) are trivially fulfilled with $\Phi=$ const and $V(\Phi)=$ const, while in our case, their solution becomes nontrivial. Introducing the new time parameter $u=\mathrm{i} \tau$ in Eq. (52) reveals that owing to $d \Phi / d u=(d \Phi / d \tau)(d \tau / d u)=-\mathrm{i} d \Phi / d \tau$, the quantity $\Phi$ becomes imaginary whence we set

$$
\Phi(\tau(u))=-\mathrm{i} \varphi(u) \quad \text { with real } \quad \varphi(u) .
$$

7 The picture obtained here (Fig. 1) is widely similar to that of Fig. 1(a) in Ref. [7]. Only the semicircle at the bottom of Vilenkin's figure does not exhibit a tip at $x=0$ as our figure what it actually should: the inverse $t(a)=H^{-1} \arccos (H a)$ of Vilenkin's solution $a(t)=H^{-1} \cos (H t)$, which can easily be obtained by putting $\gamma=2$ in Eq. (57) with (49a) (dotted curve in Fig. 1), has a tip there. Note, however, that according to Vilenkin his figure is only a symbolical representation. 


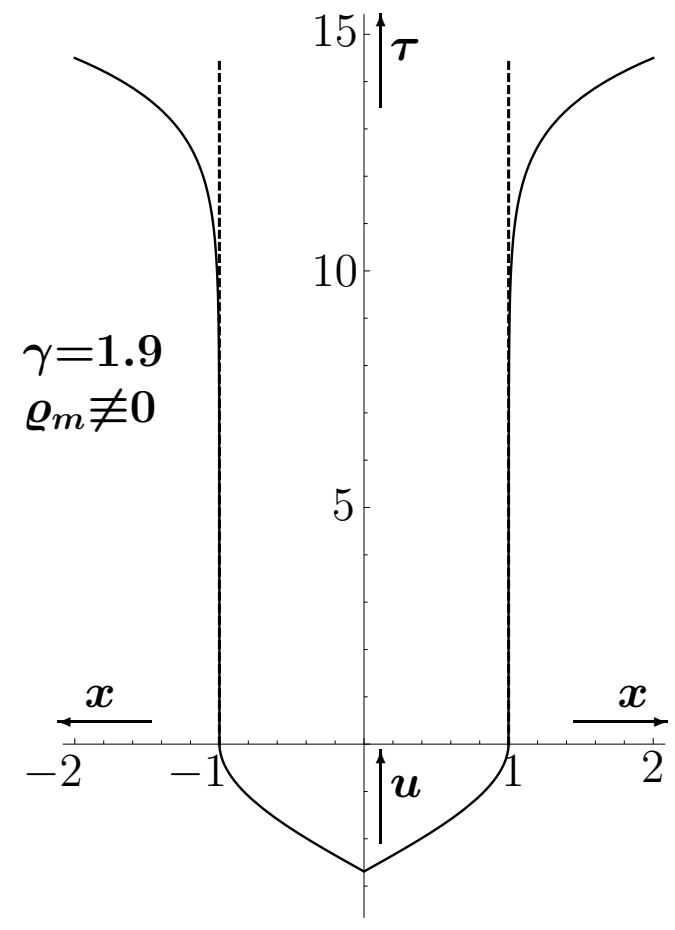

FIG. 2. Curves $\tau(x)$ and $u(x)$ in the case of simultaneous tunneling of matter, $\varrho_{m i}$ and $\varrho_{\Phi i}$ chosen according to Eqs. (47). The classical evolution, represented by the upper solid curves, starts at $\tau=0$ from a small perturbation of the equilibrium state represented by the dashed vertical lines.

With this and Eq. (56), Eq. (52) is converted into

$$
\dot{\varphi}(u)=\frac{t_{P} c^{2}}{\hbar} \sqrt{\frac{(2-\gamma) \mu \varrho_{\Phi i}}{3}} x^{\gamma / 2-1} .
$$

From this and Eq. (57) in analogy to Eq. (55) we obtain

$$
\varphi(x)=-\frac{t_{P} c^{2}}{\hbar} \sqrt{\frac{(2-\gamma) \mu \varrho_{\Phi i}}{3}} \int_{x}^{1} \frac{\xi^{\gamma / 2} d \xi}{\xi \sqrt{1-g(\xi)}} .
$$

Using $\quad \varphi(0)=-\frac{t_{P} c^{2}}{\hbar} \sqrt{\frac{(2-\gamma) \mu \varrho_{\Phi i}}{3}} J_{\gamma} \quad$ with $\quad J_{\gamma}=\int_{0}^{1} \frac{\xi^{\gamma / 2} d \xi}{\xi \sqrt{1-g(\xi)}}$

we can bring this and the result (55) into the forms

$$
\tilde{\varphi}(x)=\frac{\varphi(x)}{|\varphi(0)|}=-\frac{1}{J_{\gamma}} \int_{x}^{1} \frac{\xi^{\gamma / 2} d \xi}{\xi \sqrt{1-g(\xi)}}, \quad \tilde{\Phi}(x)=\frac{\Phi(x)}{|\varphi(0)|}=\frac{1}{J_{\gamma}} \int_{x_{g}}^{x} \frac{\xi^{\gamma / 2} d \xi}{\xi \sqrt{g(\xi)-1}},
$$

and Eq. (53) can be written as

$$
\tilde{V}(x)=\frac{V(x)}{V_{i}}=x^{\gamma-2} \quad \text { where } \quad V_{i}=V(1)=\frac{(4+\gamma) \varrho_{\Phi i} c^{2}}{6} .
$$




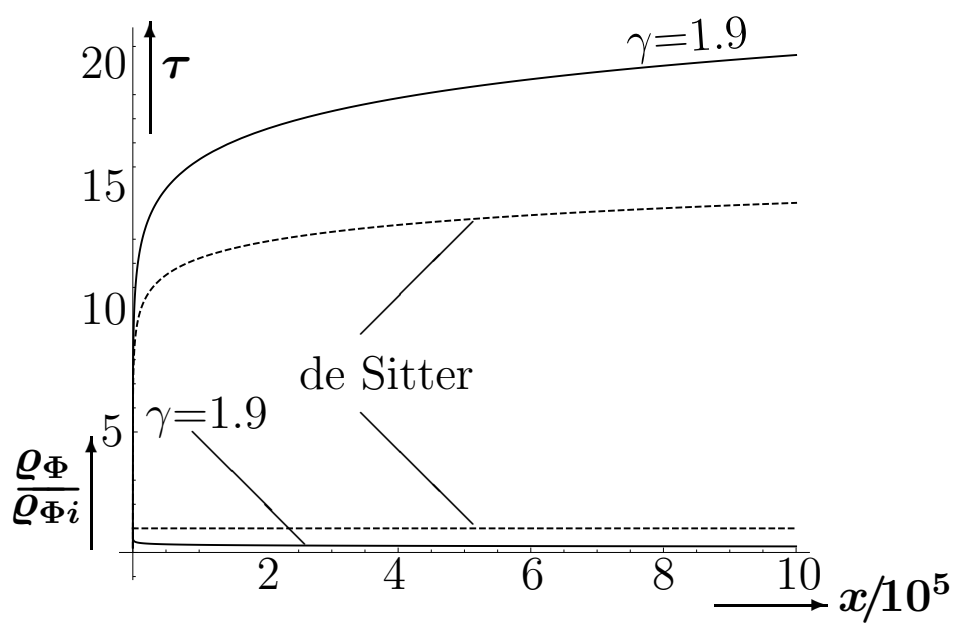

FIG. 3. Normalized age $\tau(x)$ and normalized mass density $\varrho_{\Phi}(x) / \varrho_{\Phi i}$ of the multiverse for larger values of $x$, the solid curves representing $\gamma=1.9, \varrho_{m} \equiv 0$ and the dotted ones de Sitter space $(\gamma=2)$. (The curves are obtained from Eq. (42a) and Eqs (59a).)

Eqs. (61) and (62) constitute parametric representations of the functions $\tilde{V}(\tilde{\varphi})$ and $\tilde{V}(\tilde{\Phi})$ shown in Fig. 4 for the cases with and without matter. When in the latter case, the ansatz $\varrho_{\Phi} \sim x^{\gamma-2}$ is retained within the tunneling regime $x<1$, then $\tilde{V}(\tilde{\varphi})$ diverges together with $\varrho_{\Phi}$ at $x=0$, and the shape of the combined curves $\tilde{V}(\tilde{\varphi})$ and $\tilde{V}(\tilde{\Phi})$ resembles that of a ski jump with vanishing slope at $x=0$. Introducing a cutoff by keeping $\varrho_{\Phi}$ at the value $\varrho_{\Phi i}$ in the whole tunneling regime leads to $V(\varphi)=V_{i}=$ const or $\tilde{V}(\tilde{\varphi})=1$, respectively (dashed curve). In the case $\varrho_{m i} \neq 0$, regularized according to Eqs. (58) in the quantum regime, the form of the curve $\tilde{V}(\tilde{\varphi})$ is almost exactly the same as without matter there, while the perturbational solution $\tilde{V}(\tilde{\Phi})$ in the classical regime remains much longer in the neighborhood of the (unstable) equilibrium.

\section{TAKING INTO ACCOUNT SUBUNIVERSES}

\section{A. Relation between mass densities in our universe and its associated multiverse}

For identification of the DE presently observed in our universe with the DE of its associated multiverse, represented by the field $\Phi$, we must determine how the mass densities of the two are related. Within the spatial dimensions of our universe or comparable subuniverses (coordinate system $S_{s}$ with time $t$, scale factor $a$ and mass density $\varrho_{s \Phi}$ of the field $\Phi$ ), which 


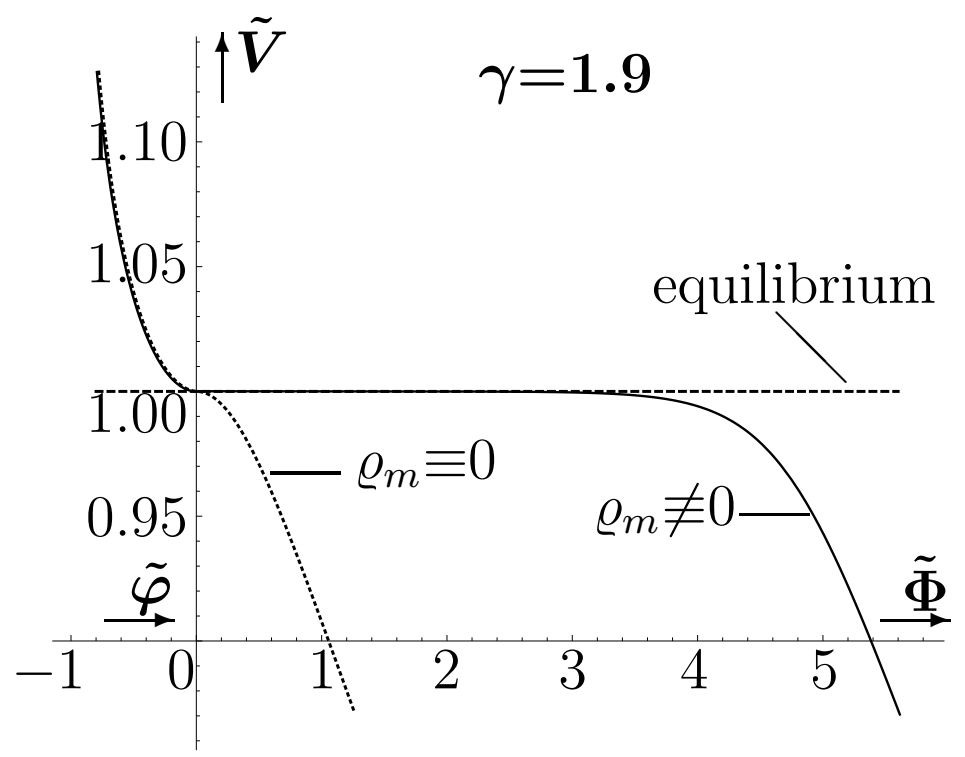

FIG. 4. $\tilde{V}(\tilde{\varphi})$ is the normalized potential $V$ in the quantum regime (to the left of the $\tilde{V}$-axis), and $\tilde{V}(\tilde{\Phi})$ is the potential in the classical regime (to the right of the $\tilde{V}$-axis), shown for the case without matter (dotted curves) and with matter (solid curves), $\varrho_{m i}$ being chosen according to Eq. (47a). On the dashed horizontal line, matter and $\Phi$-field are in an unstable equilibrium. For the solid curve on the right representing a deviation from equilibrium, we have chosen $n=3$ in Eq. (47a), $x_{g}=2$ in Eq. (61b), $\tilde{V}$ according to Eq. (62a) (slightly below 1 ) at an $x$ slightly above 1, and added an integration constant on the right hand side of Eq. (61b) such that $\tilde{\varphi}$ and $\tilde{\Phi}$ connect continuously at their respective zero.

in relation to the associated multiverse are very small, the effects of the spatial curvature are negligible (see Eq. (38)) so that the square of the line element can be written as

$$
d s_{s}^{2}=c^{2} d t^{2}-a^{2}(t) d r^{2}-a^{2}(t) r^{2} d \Omega \quad \text { with } \quad d \Omega=d \vartheta^{2}+\sin ^{2} \vartheta d \varphi^{2} .
$$

In the $\Phi$-multiverse (system $S_{M}$ ) we have

$$
d s_{M}^{2}=c^{2} d T^{2}-A^{2}(T) d \chi^{2}-A^{2}(T) \sin ^{2} \chi d \Omega^{\prime} \quad \text { with } \quad d \Omega^{\prime}=d \vartheta^{\prime 2}+\sin ^{2} \vartheta^{\prime} d \varphi^{\prime 2} .
$$

The main question is how the density $\varrho_{\Phi}$ of the $\Phi$-multiverse enters the equations valid in our universe and vice versa. The usual general relativistic equations for the transformation between different coordinate systems refer to the same physical situation. We construct such a situation by conceptually removing the matter density $\varrho_{s m}$ from our universe, this way transferring the total mass density $\varrho_{s m}+\varrho_{s \Phi}$ into $\varrho_{s \Phi}$; simultaneously we maintain the 
coordinates $t, r, \vartheta$ and $\varphi$ of the universe. Now, we consider the same situation from the coordinate system $T, \chi, \vartheta^{\prime}$ and $\varphi^{\prime}$ of the $\Phi$-multiverse where $\varrho_{\Phi}=\varrho_{\Phi}(T)$. Thereby we assume that the origins of the two systems coincide, whence for symmetry reasons the angles $\vartheta$ and $\varphi$ can also be arranged to coincide with $\vartheta^{\prime}$ and $\varphi^{\prime}$ respectively, i.e.

$$
\vartheta^{\prime}=\vartheta, \quad \varphi^{\prime}=\varphi
$$

Thus, the mass density $\varrho_{\Phi}(T)$ of the $\Phi$-multiverse will in general appear as $\varrho_{s \Phi}(t, r)$ in our universe.

The mass density $\varrho$ is a constituent of the energy-momentum tensor $T_{\mu \nu}=\varrho U_{\mu} U_{\nu}$ and therefore transforms according to

$$
\varrho^{\prime} U_{\mu}^{\prime} U_{\nu}^{\prime}=T_{\mu \nu}^{\prime}=T_{\alpha \beta} \frac{\partial x^{\alpha}}{\partial x^{\prime \mu}} \frac{\partial x^{\beta}}{\partial x^{\prime \nu}}=\varrho U_{\alpha} U_{\beta} \frac{\partial x^{\alpha}}{\partial x^{\prime \mu}} \frac{\partial x^{\beta}}{\partial x^{\prime \nu}}
$$

Considering $x^{\alpha}$ as the coordinates of $S_{s}$ (our universe) and $x^{\prime \mu}$ as those of $S_{M}$, and identifying $\varrho$ and $\varrho^{\prime}$ with the rest densities (whence $U_{0}=U_{0}^{\prime}=c$ and $U_{k}=U_{k}^{\prime}=0$ for $k=1,2,3$ ), from this we get

$$
\varrho_{s \Phi}=\varrho_{\Phi} /\left(\frac{\partial t}{\partial T}\right)^{2} .
$$

$\varrho_{s \Phi}$ must depend on $t$ only and does it, when $T=T(t)$. On the special assumption

$$
T=T_{s i}+t
$$

with $T_{s i}=$ time of creation of the subuniverse, we finally have

$$
\varrho_{s \Phi}(t)=\varrho_{\Phi}(T) \quad \text { for } \quad T=T_{s i}+t .
$$

According to Eqs. (63) and (64), $t$ and $T$ are metric times measured with identical standard clocks. Eq. (67) exhibits the space independence of $\varrho_{s \Phi}$ demanded in requirement 2 of the Introduction.

\section{B. Influence of the associated multiverse on the evolution of our universe}

According to our model, $\Phi$-multiverses provide the background for the creation of subuniverses which for their part evolve from appropriate fluctuations of independent inflatons physically different from $\Phi$. Now, we turn to the question how $\Phi$ affects the evolution of subuniverses, considering our universe as a typical example. Already, shortly after its creation, 
the primordial matter content of the multiverse is so much diluted that, as in Section II A, it can be completely neglected. During the inflation of our universe, also $\varrho_{s \Phi}$ is so much smaller than the mass density of its inflaton, that it can be neglected as well, and according to table I, inside the universe, the curvature of the multiverse can be omitted all the more. In consequence, the early evolution of our universe proceeds according to usual concepts, must not be reviewed here, and we can restrict our consideration to the period after inflation and photon decoupling. In this era, matter obeys the equation $\varrho_{s m}=\varrho_{s m 0} a_{0}^{3} / a^{3}(t)$. The influence of the $\Phi$-multiverse on our universe consists in imprinting $\Phi$ on it as an external field with prescribed dynamics given by Eq. (39a) together with Eq. (35). With neglect of the curvature term $-c^{2} a^{2} / A^{2}$, the Friedmann-Lemaitre equation of our universe becomes

$$
\dot{a}^{2}(t)=\frac{8 \pi G}{3}\left(\varrho_{s m}+\varrho_{\phi}\right) a^{2}=\frac{8 \pi G}{3}\left(\frac{\varrho_{s m 0} a_{0}^{3}}{a}+\varrho_{\Phi 0} x^{\gamma-2} a^{2}\right)
$$

or

$$
\dot{y}^{2}(t)=\frac{8 \pi G}{3}\left(\frac{\varrho_{s m 0}}{y}+\varrho_{\Phi 0} x^{\gamma-2} y^{2}\right) \quad \text { with } \quad y=\frac{a}{a_{0}} .
$$

For calculating the acceleration $\ddot{y}(t)$ at the present time, $t_{0}$ in our universe and $T_{0}=T_{s i}+t_{0}$ in the multiverse, we differentiate the last equation with respect to $t$ and then divide it by $2 \dot{y}(t)$. Using the approximation

$$
\frac{d x}{d t}=\dot{x}(\tau) \frac{d \tau}{d T} \frac{d T}{d t}=\frac{0.826 x^{\gamma / 2}}{t_{H 0}}=0.826 H_{0} x^{\gamma / 2},
$$

following from Eqs. (30), (4a), (5) and (66), furthermore $\dot{y}_{0}=\dot{y}\left(t_{0}\right)=\dot{a}\left(t_{0}\right) / a_{0}=H_{0}, x_{0}=y_{0}=1$ and $H_{0}^{2}=8 \pi G\left(\varrho_{s m 0}+\varrho_{\Phi 0}\right) / 3=8 \pi G \varrho_{c 0} / 3$, and finally using $\varrho_{s m 0}=0.317 \varrho_{c 0}$ and Eq. (8), after some rearrangement, we obtain

$$
\ddot{y}_{0}=\ddot{y}\left(t_{0}\right)=H_{0}^{2}[0.525-0.282(2-\gamma)] .
$$

Positive acceleration is achieved for $\gamma>0.14$. For later purposes also the present values of $\dddot{y}$ and the time derivative of the deceleration parameter $q=-\ddot{a} /\left(H^{2} a\right)=-\ddot{y} y / \dot{y}$ (present value $\left.q_{0}=-\ddot{y}_{0} / H_{0}\right)$ are noted down, which can be derived in a similar way:

$$
\dddot{y}_{0}=H_{0}^{3}[1-(2-\gamma)(0.441+0.270 \gamma)], \quad \dot{q}_{0}=-H_{0}[9.74-(2-\gamma)(0.449+0.111 \gamma)] .
$$

In table I for several values of $\gamma$, the acceleration $\ddot{y}_{0}$ is entered in multiples of the acceleration

$$
\ddot{y}_{\Lambda}=\frac{\ddot{a}_{\Lambda}}{a_{0}}=\frac{4 \pi G}{3}\left(2 \varrho_{\Lambda}-\varrho_{s m 0}\right)=0.525 H_{0}^{2}
$$


induced by a cosmological constant $\Lambda$ corresponding to a mass density $\varrho_{\Lambda}=\varrho_{\Phi 0}$. In order that the present acceleration by the field $\Phi$ is comparable with that by $\Lambda$, we must have $\gamma \gtrsim 1.95$ and correspondingly high values of $\zeta$ according to table I. In units of $t_{H 0}$, the age of our universe is $\tau_{u}=0.98$. With this and Eq. (34b), we get from Eq. (35) for the expansion $x_{s}$ of the multiverse at the start of our universe

$$
x_{s}=\left(\frac{\tau_{s}}{\tau_{0}}\right)^{\frac{1}{1-\gamma / 2}}=\left(\frac{\tau_{0}-0.98}{\tau_{0}}\right)^{\frac{1}{1-\gamma / 2}}=\left(1-\frac{0.813}{0.83 \tau_{0}}\right)^{0.83 \tau_{0}}<\mathrm{e}^{-0.813} \approx 0.443 .
$$

This is listed in table I for several values of $\gamma$ and shows that during the lifetime of our universe, the expansion of the multiverse more than doubles. For the density $\varrho_{\Phi h}$ at half of its lifetime, $\tau_{h}=\tau_{0}-0.49$, from Eqs. (28a) and (35), we get

$$
\frac{\varrho_{\Phi h}}{\varrho_{\Phi 0}}=x_{h}^{\gamma-2}=\left(\frac{\tau_{h}}{\tau_{0}}\right)^{\frac{\gamma-2}{1-\gamma / 2}}=\left(\frac{\tau_{0}}{\tau_{0}-0.49}\right)^{2} .
$$

This is again shown in table I; for $\gamma \gtrsim 1.95$, the density $\varrho_{\Phi}$ changes so little that its action on the dynamics of our universe is almost the same as that of a cosmological constant. In particular, it causes the same acceleration of the present expansion, and the change from deceleration to acceleration occurs at about half the life time of our universe.

\section{Influence of the subuniverses on the evolution of the multiverse}

So far, the evolution of the multiverse was treated without regard to its subuniverses. To make up for this, we determine the velocity at which our universe expands into its associated multiverse. Since we are only interested in the bulk motion, we employ a very simple model of the boundary between the two, assuming a discontinuous jump of $\varrho_{s m}$ from a uniform inside value to zero outside. Furthermore, we assume again that origin and angles of the spatial coordinates of multiverse and universe coincide.

In the coordinate system $S_{s}$ of our universe, the position of the boundary is $r=r_{b}=$ const. For a fixed point on it, $d r=d \vartheta=d \varphi=0$ whence from Eq. (63) we get

$$
d s_{s}^{2}=c^{2} d t^{2}
$$

In the system $S_{M}$ of the multiverse, the position $\chi_{b}(T)$ of this point will in general change with $T$, whence with $d \vartheta=d \varphi=0$, we obtain from Eq. (64)

$$
d s_{M}^{2}=c^{2} d T^{2}-A^{2}(T) d \chi_{b}^{2}=\left[c^{2}-A^{2}(T) \dot{\chi}_{b}^{2}(T)\right] d T^{2} .
$$


The invariance $d s_{s}=d s_{M}$ of the line element with respect to coordinate transformations leads to

$$
\frac{d t}{d T}=\sqrt{1-v^{2} / c^{2}} \quad \text { with } \quad v=A(T) \dot{\chi}_{b}(T) .
$$

The following calculations serve for the evaluation of $v$ or $\dot{\chi}_{b}(T)$ respectively. For the distance $d l$ of two neighboring points on the boundary $r=r_{b}$ which differ only in $\vartheta$ (i.e. $d t=0$ and $d r=d \varphi=0$ ), we get from Eq. (63) $d l_{s}^{2}=-d s_{s}^{2}=a^{2}(t) r_{b}^{2} d \vartheta^{2}$ in $S_{s}$, and from Eqs. (64)-(65) we get $d l_{M}^{2}=-d s_{M}^{2}=A^{2}(T) \sin ^{2} \chi_{b} d \vartheta^{2}$ in $S_{M}$, because according to Eq. (70) or $d T=d t / \sqrt{1-v^{2} / c^{2}}$ we have $d T=0$ for $d t=0$ and $d \chi_{b}=\dot{\chi}_{b}(T) d T=0$. In this case the invariance of the line element, $d l_{s}=d s_{M}$, leads to

$$
a(t) r_{b}=A(T) \chi_{b}(T)
$$

where $A \sin \chi_{b}$ was replaced by $A \chi_{b} \operatorname{since~} \sin \chi_{b}=r_{b} a / A$ is extremely small. Differentiating Eq. (71) with respect to $T$ and then inserting Eqs. (70) leads to

$$
\dot{a}(t) r_{b} \sqrt{1-v^{2} / c^{2}}=\dot{A}(T) \chi_{b}+v
$$

and resolving this equation with respect to $v$ eventually yields

$$
v=\frac{\dot{a}(t) r_{b} \sqrt{1+\left(\dot{a}(t) r_{b} / c\right)^{2}-\left(\dot{A}(T) \chi_{b} / c\right)^{2}}-\dot{A}(T) \chi_{b}}{1+\left(\dot{a}(t) r_{b} / c\right)^{2}} .
$$

(A second solution with a minus sign in front of the root term was discarded since for $a(t) \rightarrow 0$ or equivalently $\chi_{b} \rightarrow 0$ (see Eq. (71)), Eq. (72) requires $v \rightarrow \dot{a}(t) r_{b} \sqrt{1-v^{2} / c^{2}}>0$.) For evaluating $v$ at the present time $t_{0}$, we use

$$
\dot{a}\left(t_{0}\right) r_{b}=H_{0} a_{0} r_{b}=\frac{a_{0} r_{b}}{t_{H 0}}=\frac{2 R}{t_{H 0}}=3.36 \mathrm{c} .
$$

Thereby, for the present distance of the outer boundary of the universe,

$$
a_{0} r_{b}=2 R
$$

was assumed, so that inhomogeneities propagating from outer regions inwards cannot have spoiled the homogeneity and isotropy inside the observable universe $a_{0} r \leq R$ (see p. 231 of Ref. [9]); furthermore, Eqs. (5) and (10) were used. From Eqs. (4), with the approximation $\dot{x}(\tau)=0.826 x^{\gamma / 2}$ of Eq. (30), and with $x=1$ we get

$$
\dot{A}\left(T_{0}\right)=\left.A_{0} \dot{x}(\tau) \frac{d \tau}{d T}\right|_{T_{0}}=\frac{0.826 A_{0}}{t_{H 0}},
$$


and from Eq. (75) with Eqs. (71) and (16b), we obtain

$$
2 R=A_{0} \chi_{b 0}=\zeta R \chi_{b 0} \quad \text { or } \quad \chi_{b 0}=\frac{2}{\zeta}
$$

where $\chi_{b 0}=\chi_{b}\left(T_{0}\right)$. Combining Eqs. (76) and (77a), and using Eqs. (5) and (10) yields

$$
\dot{A}\left(T_{0}\right) \chi_{b 0}=\frac{0.826 A_{0} \chi_{b 0}}{t_{H 0}}=\frac{1.65 R}{t_{H 0}}=2.77 \mathrm{c} .
$$

Inserting this and the result (74) in Eqs. (73) and (70a), we finally obtain

$$
v\left(T_{0}\right)=0.36 c,\left.\quad \frac{d t}{d T}\right|_{t_{0}}=0.93
$$

and

$$
\dot{\chi}_{b}\left(T_{0}\right)=\frac{v\left(T_{0}\right)}{A_{0}}=\frac{0.36 c}{\zeta R}=\frac{0.48 \cdot 10^{-18} \mathrm{~s}^{-1}}{\zeta} .
$$

According to Eq. (77b) the present value of $\chi_{b 0}$ is extremely small since according to table I, $\zeta$ is extremely large for $\gamma \gtrsim 1.95$. At the present rate of change, the time required for doubling $\chi_{b 0}=2 / \zeta$ is

$$
\Delta T \approx \frac{\Delta \chi}{\dot{\chi}\left(T_{0}\right)}=\frac{2 / \zeta}{0.48 \cdot 10^{-18} \mathrm{~s}^{-1} / \zeta}=4.17 \cdot 10^{18} \mathrm{~s}=9.5 t_{H 0} .
$$

After this time, the matter density $\varrho_{s m}$ of our universe has already for a long time become so small that it can completely be neglected. In consequence, the volume occupied by it can be treated like the empty regions of the multiverse and expand like these as treated in Section II.

The regions of the multiverse not occupied by subuniverses are not influenced by the latter, because the gravitational field created by them cannot extend beyond their boundary: This is essentially due to Birkhoff's theorem (time-independence of all metric coefficients in the vacuum surrounding a spherically symmetric mass or energy distribution, see Ref. [16] or, e.g. Ref. [17], in combination with the fact that the gravitational field of the subuniverse was zero outside of it before its emergence). ${ }^{8}$ In consequence the unoccupied regions evolve like the corresponding regions of a $\Phi$-multiverse, which is permanently devoid of matter as treated in Section II.

\footnotetext{
${ }^{8}$ Deviating from the requirements of Birkhoff's theorem, the universe is not surrounded by vacuum but by the field $\Phi$. The theorem is valid, however, when in a first step, the field $\Phi$ is removed from the surroundings. Nothing changes within the universe when the field is brought back to them in a second step, because a homogeneous mass distribution exhibits no gravitational field within a spherical cavity. (Homogeneous mass distribution can be assumed on large scales since the probability for the emergence of subuniverses can be assumed to be space- and time-independent.) Since there is no action on the universe, there is no reaction on the surroundings.
} 
According to what was said above, after some time, the regions occupied by subuniverses behave essentially like unoccupied regions. Therefore, the evolution of the all-embracing space-time can be treated without regard to subuniverses for all times. (We exclude the possibility that the birth rate of subuniverses would be so high and pack them so densely that they would collide and merge in a stadium when their density cannot yet be neglected.)

\section{DISCUSSION OF COSMOLOGICAL PROBLEMS, INITIAL CONDITIONS AND OBSERVATIONAL CONSTRAINTS}

It can be taken from table I that for all parameter values $\gamma$, the (normalized) radius $\zeta=A / R$ of the multiverse is very large. According to Eqs. (36) and (37), $\zeta$ and $\zeta_{m}$ grow exponentially with the present (normalized) age $\tau_{0}$ of the multiverse. Already, at the low age $\tau_{0}=3.2$, we have $\zeta=10^{100}$ and an accordingly small curvature. Thus, for practically all values $\gamma$, the curvature of the space which our universe lives in, is immeasurably small. This means that the flatness problem of our universe resolves quite naturally by simply not existing.

The phenomenon of dark energy involves two puzzling problems: Why is its presently observed density $\varrho_{\Phi 0}$ so small, and why does it almost coincide with the present density $\varrho_{m 0}$ of matter? To examine these questions within the scope of the current model, using Eqs. (8), (17a), (19), (20) and (47b), and restricting ourselves to the cases $\varrho_{m i}=0$ and $\varrho_{m i}$ given by Eq. (47a), at first, we calculate

$$
\varrho_{\Phi i}=\left\{\begin{array}{lll}
\varrho_{i} & \text { for } & \varrho_{m i}=0 \\
\varrho_{i} /(1+\gamma) & \text { for } & \varrho_{m i} \neq 0, n=3 \\
2 \varrho_{i} /(2+\gamma) & \text { for } & \varrho_{m i} \neq 0, n=4
\end{array}\right\} \quad \text { with } \quad \varrho_{i}=0.98 \cdot 10^{122} \varrho_{\Phi 0}
$$

This holds for any multiverse created out of nothing and shows that initially $\varrho_{\Phi}$ has roughly the value following for quantum fluctuations from elementary particle physics with cut-off, but without renormalization and regard to symmetry breaking (see e.g. Ref. [2]). It may be pointed out here, that the initial condition (11), which is responsible for the creation out of nothing, is not only satisfied by the initial values (16a) and (19), but also by $x_{i}=\lambda l_{P} / A_{0}$ and $\varrho_{i}=3 \varrho_{P} /\left(8 \pi \lambda^{2}\right)$ with $\lambda>1$. This provides some flexibility which might be useful for adjustments concerning the initial magnitude of the multiverse or the initial densities. 
Subuniverses of earlier origin than ours had to cope with higher values $\varrho_{\Phi}>\varrho_{\Phi 0}$. In order that the galaxies of our universe could develop in such a way as we observe them, $\varrho_{\Phi}$ cannot be much larger than $\varrho_{\Phi 0}$, a factor 10 would already be too much. Thus, against higher values, the anthropic principle can be invoked. Much smaller values would, however, still be possible without much change in the appearance of our universe, of course, except for the observed acceleration of the expansion. In the framework of a multiverse with different generations of subuniverses, the present value $\varrho_{\Phi 0}=0.683 \varrho_{c 0}$ in our universe must in view of possible smaller values be considered as a matter of chance. On the other hand, the approximate coincidence with $\varrho_{m 0}$ is a consequence of this, since in a curved universe with practically vanishing spatial curvature we have $\varrho_{m 0}=\varrho_{c 0}-\varrho_{\Phi 0}=0.317 \varrho_{c 0}=0.464 \varrho_{\Phi 0}$.

The ratio $w \equiv p_{\Phi} /\left(c^{2} \varrho_{\Phi}\right)$ is usually considered as the most important quantity for the characterization of DE properties. Modeling DE by a cosmological constant yields $w=-1$. Time dependent models of DE like quintessence usually lead to a time dependent $w(t)$ which is frequently approximated by a constant value. From Eqs. (2), (28a) and (40a) for our model we get

$$
\frac{p_{\Phi}}{c^{2}}=\varrho_{\Phi}-\frac{2 V}{c^{2}}=-\frac{(1+\gamma) \varrho_{\Phi 0}}{3 x^{2-\gamma}} \quad \text { and } \quad w=-\frac{1+\gamma}{3}
$$

i.e. we obtain a time-independent value of $w$ automatically. According to Wang et al. [3], the cosmological constant model $(w=-1)$ has so far still "the best performance in fitting the current observational data" (e.g. for data obtained from Type Ia supernova observations, see Ref. [18]), while some of them "mildly favor" $w=-1-\epsilon$ with small $\epsilon>0$, a situation which corresponds to a phantom DE with a so called big rip (see e.g. page 55 of Ref. [10]).

Inserting the ansatz $\gamma=2-3 \epsilon$ with $\epsilon>0$ in Eq. (80) we obtain $w=-1+\epsilon$. Since according to Eqs. (28), $\gamma=2$ can be approached from below as closely as wanted, $\epsilon$ can be chosen arbitrarily small. This means that our model can be fitted to the current observational data just as well as the cosmological constant model. Choosing, e.g., $3 \epsilon=0.01$ or $\gamma=1.99$ yields the (normalized) radius $\zeta=9.6 \cdot 10^{12137}$ of the multiverse and a corresponding spatial curvature of the order $10^{-24276} / R^{2}$. Since $w$ differs from $w=-1$ only very little at all times, the space-time generated by the field $\Phi(T)$ approaches period by period very closely varying de Sitter spaces with different cosmological constants.

Should forthcoming observations necessitate a value $w<-1$, this would not completely disqualify the present model, but only its representation of DE by a scalar field $\Phi$ or the use of Eqs. (2)-(3), respectively. In an appropriately changed representation the big rip 
associated with $w<-1$ would imply an additional constraint, namely that it would have to happen later than now.

The time behavior of the cosmic acceleration caused by DE is, in principle, measurable and could turn out as another criterion for the usefulness of DE models. According to most theoretical models the acceleration is still increasing. However, in 2009, it was proposed for the first time, that it "may have already peaked and that we are currently witnessing its slowing down." [19] In Subsection III B, some parameters relevant in this context were calculated. For the $\gamma$-values close to 2 following from the above, the quantities $\dddot{y}_{0}$ and $\dot{q}_{0}$ specified in Eq (69) are both yielding an increase of the cosmic acceleration. For the areas of the multiverse between the subuniverses, the situation looks somewhat different. From the approximation $\dot{x}(\tau)=\alpha x^{\gamma / 2}$ with $\alpha=0.826$ and Eq. (4b), one easily obtains

$$
q=-\frac{A \ddot{A}(t)}{\dot{A}^{2}(t)}=-\frac{\gamma}{2} \approx-1 \quad \text { and } \quad \dot{q}(t)=0 .
$$

A local quantity even more closely related to the acceleration $\ddot{A}(T)$ is the specific acceleration $S=\ddot{A}(T) / A$. For this, we get

$$
S(t)=\frac{\gamma \alpha^{2} x^{\gamma-2}}{2 t_{H 0}^{2}} \quad \text { and } \quad \dot{S}(t)=-\frac{\gamma(1-\gamma / 2) \alpha^{3}}{t_{H 0}^{2} x^{3(1-\gamma / 2)}}
$$

Whereas $\dot{S}(t)$ is negative for the pure DE field of a $\Phi$-multiverse, the time derivative of corresponding quantity $s=\ddot{a}(t) / a$ in our universe is not much different from $\dddot{y}_{0}$ given by Eq. (69a) (only $0.441 \rightarrow 0.723$ ) and is positive.

Recently, a comprehensive study on the time behavior of the cosmic acceleration (CA) was performed, considering both theoretical models and observational data [20]. It was concluded that "due to the low significance, the slowing down of $\mathrm{CA}$ is still a theoretical possibility that cannot be confirmed by the current observations." Furthermore, it was found, that "a flat Universe favors an eternal CA, while a non-flat Universe prefers a slowing down CA." This is, if only partly, supported by our model: $\dot{S}_{0}=-\gamma(1-\gamma / 2) \alpha^{3} / t_{H 0}^{2}$, the present value of $\dot{S}(t)$, is zero at $\gamma=2$ and assumes its minimum $\dot{S}_{0}=-\alpha^{3} / t_{H 0}^{2}$ at $\gamma=1$. Since according to Eq. (36b) or table I, the curvature $K=1 /(R \zeta)^{2}$ of the multiverse increases with decreasing $\gamma<2$, the CA is indeed progressively slowing down with increasing spatial curvature at least in the interval $1 \leq \gamma<2$. 


\section{CONCLUSIONS}

Special models of a multiverse were analyzed whose main purpose consists in providing space and time for a multitude of subuniverses with ours among them. Specific reasons led to assuming closed space geometry with positive curvature for homogeneous and isotropic $\Phi$-multiverses which are generated by a scalar quantum field $\Phi$ and were shown to provide the background and embracing frame of the gradually emerging subuniverses. Constituting space-times of similar origin and structure, the $\Phi$-multiverses can be interpreted as generalizations of de Sitter space, period-wise approaching varying manifestations of it very closely. The scalar field $\Phi$, needed for driving an inflation-like expansion of them, is chosen such that it originates by a creation out of nothing via quantum-mechanical tunneling. Its energy density $\varrho_{\Phi} c^{2}$ can be identified directly with that of the DE in our universe, what implies that it causes the presently observed acceleration of the expansion of our universe. A further generalization results from incorporating primordial matter in the process of creation out of nothing. Through this, the starting point of spatial expansion is endowed with the properties of an unstable equilibrium, leading to an especially efficient initial homogenization of all physical quantities. Furthermore the inclusion of primordial matter can be interpreted such, that together with the ingredients also the information about their physical properties emerge from the tunneling process. In this sense of information transmission, the simple matter density $\varrho_{m}$ of the multiverse can be understood as an all-inclusive representative for all kinds of matter to appear later in subuniverses.

The mass density $\varrho_{\Phi}$ of the dark energy field $\Phi$ can be expressed as a monotonic function of the spatial curvature of the multiverse, decreasing and approaching zero simultaneously with it (Subsection II A 2). This has a significant implication: It is an important consequence of a famous principle of $\mathrm{Mach}^{9}$ (see Ref. [23] or p. 179 and p. 199 in Ref. [11]) that space and time are only meaningful in the presence of matter or energy. This is automatically satisfied by the current model, because at all finite times, the spatial curvature and with it $\varrho_{\Phi}$ is unequal zero. Furthermore, $\varrho_{\Phi}$ is an indirectly measurable quantity, and its permanent decay provides an arrow of time.

\footnotetext{
9 Mach's principle can be summarized in short as: The inertia of each mass is caused by its interaction with all other masses in the universe. In developing general relativity, Einstein was influenced by this principle. To his disappointment (see Ref. [21]), Mach's principle was no general consequence of his field equations since, e.g., flat space-time is a solution. (Einstein considered de Sitter space in the first place as an obstacle [see Postscript in Ref. [22]], and for some time, he looked for ways to rule it out.)
} 
It appears worth mentioning that the extension of solutions to imaginary values of the time and the scalar field $\Phi$ preserves their property of being exact solutions of the underlying equations. In the case of $\Phi$, an imaginary value is nothing unusual because quantum fields can even be complex. It is quite another matter, that simultaneously, the transition to a regime takes place, in which a not yet fully available quantum theory of the gravitational field would be required. Thereby $\Phi$ raises no problems again, because it is already a quantum quantity. Concerning the gravitational field represented by $A(T)$, it fits in well that the corresponding equation, (57), can be interpreted as the quasi-classical approximation to a quantum-mechanical equation. In the case of de Sitter space, this interpretation is supported by approximate solutions of the Wheeler-De-Witt equation, the Schrödinger equation for stationary wave functions of a universe or multiverse, in a so-called minisuperspace [8, 24$26]$.

Like de Sitter space, the models considered in this paper must be regarded as toy models primarily chosen because of mathematical simplicity. They are well-suited for demonstrating the feasibility of certain physical properties, but nature may well prefer other models. For this reason Section II A started with employing a more general density $\varrho_{\Phi}=\varrho_{\Phi 0} f(x)$. It was found in this context that the initial value $\varrho_{i}$ of the mass density $\varrho$ is, independent of its composition and later behavior, already fully determined by the requirement of zero initial expansion velocity, the prerequisite for quantum tunneling from nothing. According to table I, in terms of the age of our universe, the present age of the underlying space-time is not especially old, a big part of it being spent for the early evolution $(\tau(x)$ assumes the values $\tau(1)=242, \tau\left(10^{-5}\right)=228$ and $\tau\left(10^{-10}\right)=216$ for $\left.\gamma=1.99\right)$; in contrast, its present radius assumes extremely high values so that the spatial curvature lies far below measurability.

According to Section IV, by choosing $\gamma=2-3 \epsilon$ or $w=1-\epsilon$ with sufficiently small $\epsilon>0$, our model can be fitted to the current observational data as well as the cosmological constant model, which has "the best performance" in this respect. In a multiverse, all subuniverses beyond ours are observationally inaccessible, unless ours has had a collision with another one [27]. An observation of this kind would yield a direct proof of the existence of a multiverse. In future, a much more precise observational determination of $\varrho_{\Phi}(t)$ could potentially yield an indirect proof of the validity of our model (or modifications of it), the observational verification of $w \neq-1$ being a necessary precondition; the same holds for temporal changes of the cosmic acceleration, because present observations still admit no unequivocal conclusions. 


\section{Appendix A: Additional motivation for closed space geometry}

In a previous paper [28], it was shown that in uncurved open space, the recession of galaxies of our universe, usually interpreted by an expansion of space, can be explained equivalently by a motion of the cosmic substrate across radially invariable space, caused by an explosion-like big bang or by inflation. The two interpretations are not in contradiction but are related to each other by a one-to-one transformation between the specific coordinates to which each of them is restricted, co-moving FRW coordinates in the case of space expansion and "explosion coordinates" in the case of motion across radially invariable space.

In a spatially flat universe, the transition from expansion coordinates $t, r, \vartheta, \varphi$ to explosion coordinates $\tau, \rho, \vartheta, \varphi$ is accomplished by a transformation $t=t(\rho, \tau), r=r(\rho, \tau)$ such that the square of the line element,

$$
d s^{2}=c^{2} d t^{2}-a^{2}(t)\left[d r^{2}+r^{2}\left(d \vartheta^{2}+\sin ^{2} \vartheta d \varphi^{2}\right)\right]
$$

in FRW coordinates, is transformed into

$$
d s^{2}=c^{2} g_{00}(\rho, \tau) d \tau^{2}-d \rho^{2}+g_{\Omega}(\rho, \tau)\left(d \vartheta^{2}+\sin ^{2} \vartheta d \varphi^{2}\right)
$$

In expansion coordinates, the radial expansion is expressed by the time-dependence of the length element $d l_{r}=a(t) d r$, whereas in explosion coordinates, there is no radial expansion due to $d l_{\rho}=d \rho$. Inserting $d t=t_{\rho} d \rho+t_{\tau} d \tau$ and $d r=r_{\rho} d \rho+r_{\tau} d \tau$ in Eq. (A1) and comparing with Eq. (A2) leads to

$$
c^{2} t_{\rho} t_{\tau}=a^{2} r_{\rho} r_{\tau}, \quad c^{2} t_{\rho}^{2}-a^{2} r_{\rho}^{2}=-1, \quad g_{00}=t_{\tau}^{2}-a^{2} r_{\tau}^{2} / c^{2}, \quad g_{\Omega}=-a^{2} r^{2}
$$

In Ref. [28], it was shown that these equations have solutions observing the conditions $\rho=0$ and $t=\tau$ at $r=0$.

In a (closed) universe of positive curvature, the square of the line element in FRW coordinates is

$$
d s^{2}=c^{2} d t^{2}-a^{2}(t)\left[d \chi^{2}+\sin ^{2} \chi\left(d \vartheta^{2}+\sin ^{2} \vartheta d \varphi^{2}\right)\right]
$$

Since the non-angular part is exactly the same as in a flat universe (only $r$ being replaced by $\chi)$, as far as time and the radial coordinate are concerned, the transformation to explosion coordinates can be transferred without alterations. This means that in a closed universe, the transition to explosion coordinates without radial expansion is possible as well. However, 
a severe restriction must be made here: Explosion coordinates are meaningful only locally; on a global scale they would be associated with counter-streams of matter or galaxies, and, still worse, the radial extent of the universe would remain unaltered from the very beginning and have to start with extreme magnitude in order to fit in with present observations.

From this, we infer that in a closed curved multiverse, an intrinsic global expansion of space exists which cannot be transformed away and is not present in an uncurved open multiverse. This served as an additional motivation for employing closed space geometry and endowing the field $\Phi$ with intrinsic properties.

A different and even simpler approach leads to the same conclusion. In flat and infinitely extended space, a big bang with or without inflation is a local event restricted to a region that is initially extremely small but extends very rapidly. The boundary of the affected region propagates into its surroundings at the speed of light. Outside, the situation is the same as before the big bang, and there is no reason whatever for an expansion of space there. At the present time $t_{0}$, the metric radius of the region influenced by the big bang is $c t_{0}$ when judged from outside whereas it is appreciably larger when judged from inside in the system of co-moving FRW coordinates. (Already the distance of the particle horizon is $\approx 2.3 c t_{0}$.) This means, that the internal and external coordinate systems cannot be joined in a meaningful manner, and indicates, that an internal continuation of the external coordinates must exist which, as the latter ones, exhibit no radial space expansion.

\section{Appendix B: Slow roll approximation}

Slow roll means that the term $\ddot{\Phi}(T)$ on the left hand side of Eq. (3) is dominated by the friction term $\sim \dot{\Phi}(T)$, i.e. $|\ddot{\Phi}(T) /(3 H \dot{\Phi}(T))| \ll 1$. Differentiating the square of Eq. (22) with respect to $T$ and subsequently dividing it by $2 \dot{\Phi}(T)$ yields

$$
\ddot{\Phi}(T)=-\frac{\mu c^{4} \dot{A}(T)\left(\varrho_{\Phi}^{\prime}(A)+A \varrho_{\Phi}^{\prime \prime}(A)\right)}{6 \hbar^{2} \dot{\Phi}(T)}
$$

with what we get

$$
\frac{\ddot{\Phi}(T)}{3 H \dot{\Phi}(T)}=\frac{1}{6}\left(1+\frac{A \varrho_{\Phi}^{\prime \prime}(A)}{\varrho_{\Phi}^{\prime}(A)}\right) .
$$

Inserting in this $\varrho_{\Phi}(A)=\varrho_{\Phi 0} A^{\gamma-2} / A_{0}^{\gamma-2}$ from Eqs. (4a) and (28a) yields

$$
\left|\frac{\Phi(\ddot{T})}{3 H \dot{\Phi}(T)}\right|=\frac{2-\gamma}{6} \leq 10^{-2} \quad \text { for } \quad \gamma \geq 1.94 \text {. }
$$


Neglecting $\ddot{\Phi}(T)$ and using $H=\dot{A}(T) / A(T), V^{\prime}(\Phi)=\dot{V}(T) / \dot{\Phi}(T)$ as well as $\dot{V}(T) / \dot{A}(T)=V^{\prime}(A)$, from Eq. (3), we obtain

$$
\frac{3 \dot{A}(T) \dot{\Phi}(T)}{A(T)}=-\frac{\mu c^{2}}{\hbar^{2}} V^{\prime}(\Phi)=-\frac{\mu c^{2} \dot{V}(T)}{\hbar^{2} \dot{\Phi}(T)} \quad \text { or } \quad \dot{\Phi}^{2}(T)=-\frac{\mu c^{2} A V^{\prime}(A)}{3 \hbar^{2}} .
$$

With Eq. (22), the last equation becomes $V^{\prime}(A)=c^{2} \varrho_{\Phi}^{\prime}(A)$. Choosing an integration constant such that $V \rightarrow 0$ for $A \rightarrow \infty$, by integration and with $A=A_{0} x$, we finally obtain

$$
V(x)=c^{2} \varrho_{\Phi}(x)=\varrho_{\Phi 0} c^{2} x^{\gamma-2} .
$$

[1] S. Perlmutter et al., Astrophys. J. 517, 565 (1999).

[2] S. Weinberg, The Cosmological Constant Problems (talk given at Dark Matter 2000, Marina del Rey, CA), arXiv:astro-ph/0005265v1 (2000).

[3] S. Wang, Y. Wang and M. Li, Holographic Dark Energy, to appear in Physics Reports. arXiv:1612.00345v1 [astro-ph.CO] (2016).

[4] G. W. Gibbons, S. W. Hawking, S. T. C. Siklos, eds., "Natural Inflation". The Very Early Universe (Cambridge University Press 1983) p. 251.

[5] A. Vilenkin, Phys. Rev. D 27, 2848 (1983).

[6] A. Linde, Prospects of Inflation, (Physica Scripta Online 2004). http://arXiv:hep-th/0402051

[7] A. Vilenkin, Phys. Lett. B 117, 25 (1982).

[8] A. Vilenkin, Nucl. Phys. B 252, 141 (1985).

[9] V. Mukhanov, Physical Foundations of Cosmology (Cambridge University Press, Cambridge 2005).

[10] S. Weinberg, Cosmology (Oxford University Press 2008) p. 209.

[11] E. Rebhan, Theoretische Physik: Relativitätstheorie and Kosmologie (Spektrum Akad. Verlag/Springer-Verlag, Berlin Heidelberg 2012) p. 385.

[12] E. Rebhan, Astron. Astrophys. 353, 1 (2000).

[13] S. Coleman, Phys. Rev. D 15, 2929 (1977).

[14] J. Hartle and S. Hawking, Physical Review D 28, 2960 (1983).

[15] J. Louko, Einstein Online 04, 1016 (2010).

http://www.einstein-online.info/spotlights/quantum_cosmo_path_integrals 
[16] G. Birkhoff, Relativity and Modern Physics (Havard University Press, Cambridge MA 1923) p. 253.

[17] S. Weinberg, Gravitation and Cosmology (John Wiley \& Sons, New York 1972) p. 337.

[18] M. Betoule et al., Astron. Astrophys. A22, 568 (2014).

[19] A. Shafieloo, V. Sanhi, A. A. Starobinsky, Phys. Rev. D 80, 101301(R) (2009).

[20] S. Wang, Y. Hu, M. Li, N. Li, Astrophys. J. 821:60 1 (2016), arXiv:1509.03461v3 [astro-ph.CO]

[21] A. Pais Subtle is the Lord. The science and life of Albert Einstein (Oxford University Press 1982) p. 287.

[22] W. de Sitter, Proc. Kon. Ned. Acad. Wet. 19, 1217 (1917).

[23] E. Mach, Die Mechanik in ihrer Entwickelung (F. A. Brockhaus, Leipzig 1883) p. 216. http://echo.mpiwg-berlin.mpg.de/ECHOdocuView?url=/mpiwg/online/permanent/ einstein _exhibition/sources/Q179XRYG/pageimg\&start=221\&pn=229 \&mode=imagepath

[24] C. M. DeWitt and J. A. Wheeler, eds., Batelle Rencontres, 1967 Lectures in Mathematics and Physics, (Benjamin, New York 1968), p. 242.

[25] B. S. DeWitt, Phys. Rev. 1601113 (1967).

[26] A. Linde, Particle Physics and Inflationary Cosmology, p. 195 (1990). http://arxiv.org/pdf/hep-th/0503203.pdf

[27] G.F.R. Ellis, J.-P. Uzan, Comptes Rendus Physique 16, 928 (2015). http://arXiv:1612.01084v1 [gr-qc]

[28] E. Rebhan, Phys. Rev. D 86, 123012 (2012). 